\title{
Regulation of Protein Structural Changes by Incorporation of a Small-Molecule Linker
}

\author{
Youngmin Kim ${ }^{1,2}$, Cheolhee Yang ${ }^{1,2} \mathbb{C}^{1}$, Tae Wu Kim ${ }^{1,2} \mathbb{D}$, Kamatchi Thamilselvan ${ }^{1,2}$, \\ Yonggwan Kim ${ }^{1,2}$ and Hyotcherl Ihee ${ }^{1,2, *(D)}$ \\ 1 Center for Nanomaterials and Chemical Reactions, Institute of Basic Science (IBS), Daejeon 34141, Korea; \\ youngmin.kim.k@gmail.com (Y.K.); eangel04@gmail.com (C.Y.); ktwk6660@gmail.com (T.W.K.); \\ nanostar.shruthi@gmail.com (K.T.); anglerlord@gmail.com (Y.K.) \\ 2 Department of Chemistry and KI for the BioCentury, Korea Advanced Institute of Science and \\ Technology (KAIST), Daejeon 34141, Korea \\ * Correspondence: hyotcherl.ihee@kaist.ac.kr; Tel.: +82-42-350-2844
}

Received: 14 October 2018; Accepted: 19 November 2018; Published: 22 November 2018

\begin{abstract}
Proteins have the potential to serve as nanomachines with well-controlled structural movements, and artificial control of their conformational changes is highly desirable for successful applications exploiting their dynamic structural characteristics. Here, we demonstrate an experimental approach for regulating the degree of conformational change in proteins by incorporating a small-molecule linker into a well-known photosensitive protein, photoactive yellow protein (PYP), which is sensitized by blue light and undergoes a photo-induced $\mathrm{N}$-terminal protrusion coupled with chromophore-isomerization-triggered conformational changes. Specifically, we introduced thiol groups into specific sites of PYP through site-directed mutagenesis and then covalently conjugated a small-molecule linker into these sites, with the expectation that the linker is likely to constrain the structural changes associated with the attached positions. To investigate the structural dynamics of PYP incorporated with the small-molecule linker (SML-PYP), we employed the combination of small-angle X-ray scattering (SAXS), transient absorption (TA) spectroscopy and experiment-restrained rigid-body molecular dynamics (MD) simulation. Our results show that SML-PYP exhibits much reduced structural changes during photo-induced signaling as compared to wild-type PYP. This demonstrates that incorporating an external molecular linker can limit photo-induced structural dynamics of the protein and may be used as a strategy for fine control of protein structural dynamics in nanomachines.
\end{abstract}

Keywords: photoactive yellow protein; small-angle X-ray scattering; chemical modification; protein structural dynamics; regulated conformational change

\section{Introduction}

Various nanomachines based on small-molecule moieties have been designed and synthesized to control structural changes and mimic numerous mechanical movements at atomic-length scales [1,2]. However, the nanomachines composed of small organic compounds may not be suitable for applications in living organisms because of their potential toxicity. In this regard, nanomachines composed of proteins having low toxicity have a potential to serve as a good alternative. However, controlling the structural changes of proteins and manipulating motion via structural regulation of proteins in molecular nanomachines are challenging, since proteins are complicated macromolecules with many levels of structural organization from the primary structure (a sequence of amino acid residues) to the quaternary structure (interaction of protein subunits). In this regard, one of the 
important challenges in developing protein-based nanomachines is to achieve fine control of the mechanical motions within proteins.

It has been reported that the structural changes of photo-responsive organic molecules bound to photo-insensitive proteins can induce subsequent structural changes in the protein. In this way, photo-insensitive proteins can be turned into photoactive proteins. A well-known example is the control of protein structural changes using azo-benzene derivatives [3]. In the case of a protein nanocage [4], an ATP-driven chaperonin was turned into a light-driven cage by binding the azobenzene-based linkers. These studies have focused on inducing structural changes of proteins using photo-switchable small molecules. In this work, we investigated a reverse version of this approach. In other words, we chose a protein known to undergo a large conformational change and attached a non-photoactive small-molecule linker to the protein to limit the extensive structural change. We aimed at restricting pre-existing structural changes or scaling down structural changes to achieve a fine control of structural changes, rather than creating new structural changes. Particularly, we investigated the possibility and the mechanism of regulating the degree of conformational change by mutations and intramolecular conjugations using a linker molecule.

For this purpose, we used photoactive yellow protein (PYP). PYP, which has $p$-coumaric acid $(p \mathrm{CA})$ as the chromophore, is an attractive model system for understanding photoreception and the subsequent signal transduction process responsible for the negative phototaxis in Halorhodospira halophila [5]. Upon the absorption of blue light, PYP undergoes a volume-conserving trans-cis isomerization of the $p \mathrm{CA}$ chromophore, which leads to a significant conformational change of the protein. This photoreaction of PYP is accompanied by changes in the absorption spectra, refractive indices, ion complexation, and electrochemical properties [6-12]. The ground state of wild-type PYP, known as $\mathrm{pG}$, has a globular protein conformation with the trans configuration of $p C A$ chromophore, whereas a light-activated state known as $\mathrm{pB}$ (or $\mathrm{pB}_{2}$ ) or $\mathrm{I}_{2}$ has a relatively expanded conformation including the protrusion of the $\mathrm{N}$-terminus and the cis configuration of the chromophore. The photoreaction of PYP involves the formation of the light-activated state populated in the millisecond time region and the relaxation of the light-activated state to the ground state takes place within milliseconds to minutes. Since sensitization with blue light in PYP causes a global conformational change and such a photoreaction is photo-reversible, we used PYP as a target system in order to demonstrate the new approach for regulating global structural changes in proteins.

We employed the incorporation of a chemical reagent, acting as a bridge connecting the protein moieties, to the modified PYP. To introduce a small-molecule linker into PYP, two amino acid residues of wild-type PYP were mutated into cysteine residues with thiol group side chains. Two thiol groups were covalently conjugated with the ends of a thiol-specific cross-linker. When PYP absorbs blue light, the cross-linker would prevent the protrusion of the N-terminus and thus the structural change of PYP can be restricted as shown in Figure 1.

To investigate the effect of the molecular linker on the N-terminus of PYP with respect to the protein conformation, we used small-angle X-ray scattering (SAXS) combined with systematic structural analysis based on experiment-restrained rigid-body molecular dynamics (MD) simulation to determine the protein shape, size, and conformational differences between the states with and without blue light irradiation and transient absorption (TA) spectroscopy to analyze the kinetics. We analyzed the conformational changes of PYP induced by conjugation of the cross-linker and the conformational changes of the modified PYP induced by irradiation. 
a

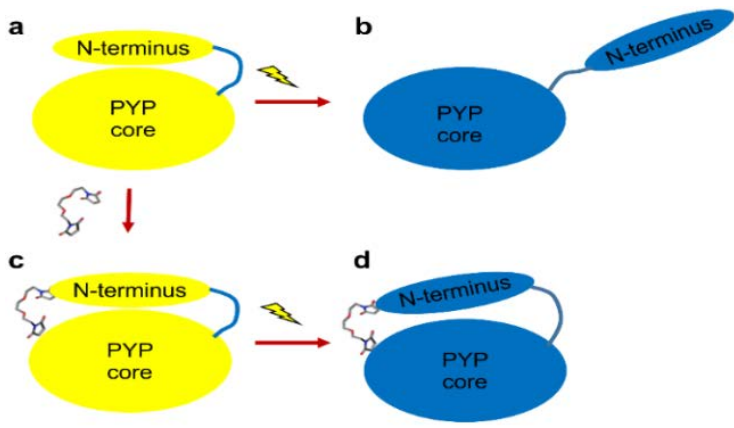

Figure 1. Schematic representation of the expected conformational change in the chemically modified photoactive yellow protein (PYP). The chemically modified protein includes the small-molecule linker (stick) covalently conjugated with the thiol groups (red-color) at the 7th and 91st residues. Wild-type PYP undergoes an N-terminal protrusion upon blue light excitation $(\mathbf{a} \rightarrow \mathbf{b})$. In the chemically modified PYP (SML-PYP), the small-molecule linker plays a critical role in regulating the degree of structural change in the photoreaction of PYP, thereby limiting the N-terminus protrusion $(\mathbf{c} \rightarrow \mathbf{d})$.

\section{Results}

\subsection{Sample Characterization}

We considered the following points when we chose the two positions at which cysteine mutations could be introduced to conjugate the small linker molecule. Since our goal is to restrict the photoinduced N-terminus movement of PYP, one of the positions should be located at the N-terminus. For this position, we chose the 7th (glycine) and 13th (asparagine) positions considering the minimal interference with other residues and the side chain direction pointing toward the protein body. For the other position in the protein body, we chose the 91st (methionine) and 113th (leucine) positions considering the minimal interference and the side chain direction pointing toward the N-terminus. Another consideration at this point was that the selected position at the N-terminus and the position at the protein body should be separated by a distance similar to or shorter than the length of the small-molecule linker. We generated and tested various cysteine-mutated PYPs with the selected mutation positions and found that only the mutant residues at the 7th (glycine) and 91st (methionine) positions were successfully conjugated with the 1,8-bismaleimidodiethyleneglycol (BM(PEG) $\left.)_{2}\right)$ linker (see Figure S1). Although the length $(\sim 14 \AA)$ of $\mathrm{BM}(\mathrm{PEG})_{2}$ is longer than the distance $(\sim 9 \AA)$ between the 7 th and 91st residues, the small molecule, which is flexible, was successfully conjugated between the mutated positions. This PYP conjugated with the BM(PEG) 2 linker was named SML-PYP, where SML stands for small-molecule linker. After incorporating the cross-linker to the PYP mutants, the samples were analyzed using UV-vis absorption spectroscopy and matrix-assisted laser desorption/ionizaiton time-of-flight (MALDI/TOF) spectrometry. The UV-vis absorption spectrum of SML-PYP is almost the same as the previously reported absorption spectrum of wild-type PYP (Figure S2), indicating that the environment around the chromophore binding pocket is not affected by the double mutation and the incorporation of the linker molecule. Based on the results from the measurement of MALDI/TOF, we confirmed that the molecular weight of SML-PYP is equal to the sum of the molecular weights of the wild-type PYP and the linker molecule (Figure S3). Moreover, Thiol Fluorescent Detection Kit (Invitrogen ${ }^{\mathrm{TM}}$ ) data showed almost no free thiols in the sample solution of SML-PYP (Figure S4), implying that most of the side chains of cysteine in the PYP mutant formed a thioether bond with the linker. We also measured the CD (circular dichroism) spectrum of SML-PYP to check the structural stability (Figure S5).

\subsection{Transient Absorption (TA) Spectroscopy}

From the fitting result of TA data, four-time constants, $\tau_{1}, \tau_{2}, \tau_{3}$, and $\tau_{4}$, were determined (Table 1 ). TA data of wild-type PYP and SML-PYP exhibited similar spectra and kinetics (Figure 2 and Table 1), 
suggesting that the conjugation of the linker and the double mutation of PYP do not affect its overall kinetics. The photocycle of PYP has been explained by two kinetic models, which are a sequential model (Figure 2d) [13-15] and a parallel model (Figure 2e) [11,16,17]. In the photocycle of PYP, two red-shifted intermediates $\left(\mathrm{pR}_{1}\right.$ and $\left.\mathrm{pR}_{2}\right)$ and two blue-shifted intermediates $\left(\mathrm{pB}_{1}\right.$ and $\left.\mathrm{pB}_{2}\right)$ compared to the spectrum of the ground state $(\mathrm{pG})$ are formed after blue light irradiation. Based on the sequential model, $\tau_{1}$ and $\tau_{2}$ correspond to the transition from $p R_{1}$ to $p R_{2}$ and from $p R_{2}$ to $p B 1$, respectively. Based on the parallel model, $\tau_{1}$ and $\tau_{2}$ correspond to the transition from $\mathrm{pR}_{1}$ to $\mathrm{pB}_{1}$ and from $\mathrm{pR} \mathrm{R}_{2}$ to $\mathrm{pB}_{1}$, respectively. In both kinetic models, $\tau_{3}$ and $\tau_{4}$ can be assigned as the transition from $\mathrm{pB}_{1}$ to $\mathrm{pB}_{2}$ and dark recovery from $\mathrm{pB}_{2}$ state to $\mathrm{pG}$ state, respectively. Unlike other transitions, the formation of $\mathrm{pB}_{2}\left(\tau_{3}\right)$ of SML-PYP is almost two times faster than that of wild-type PYP (Table 1). The $\mathrm{pB}_{2}$ state of wild-type PYP is a putative signaling state of PYP and shows the largest structural changes compared to the $\mathrm{pG}$ state during the photocycle of PYP. Hence, the accelerated formation of $\mathrm{pB}_{2}$ of SML-PYP might indicate that the structural changes of SML-PYP are regulated by the cross-linker.
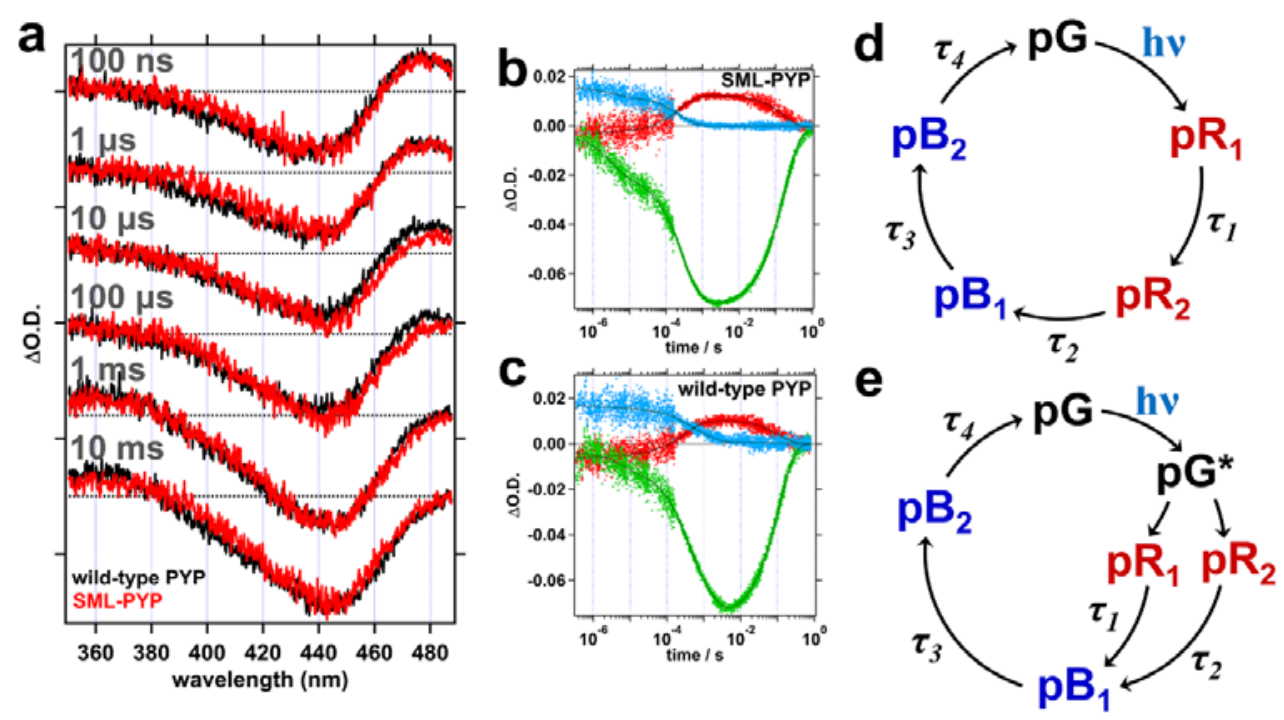

Figure 2. (a) Transient Absorption (TA) spectra of SML-PYP (red) are compared with those of wild-type PYP (black). Time delays for the TA spectra are (from top to bottom): $100 \mathrm{~ns}, 1 \mu \mathrm{s}, 10 \mu \mathrm{s}, 100 \mu \mathrm{s}, 1 \mathrm{~ms}$, and $10 \mathrm{~ms} . \Delta$ O.D. stands for the optical density change and the spectra are vertically offset for clear presentation. (b,c) Temporal profiles of TA signal for (b) SML-PYP and (c) wild-type PYP measured at the probe wavelengths of 380 (red), 465 (green), and $494 \mathrm{~nm}$ (blue) are shown. The decay profiles of each protein were globally fit by the sum of exponential functions consisting of four exponentials and an offset. The fit lines are shown in black dotted lines. The time constants determined by the fit are listed in Table 1. (d,e) Schematic illustrations of (d) the sequential kinetic model and (e) the parallel kinetic model for the photocycle of PYP. hv indicates the optical excitation.

Table 1. Time constants of SML-PYP and wild-type PYP determined by the fitting of TA data.

\begin{tabular}{ccc}
\hline Fitting Results & SML-PYP & Wild-Type PYP \\
\hline$\tau 1(\mu \mathrm{s})$ & $3.3 \pm 1.3$ & $4.4 \pm 1.2$ \\
$\tau 2(\mu \mathrm{s})$ & $200 \pm 51$ & $210 \pm 49$ \\
$\tau 3(\mu \mathrm{s})$ & $480 \pm 140$ & $920 \pm 260$ \\
$\tau 4(\mathrm{~ms})$ & $170 \pm 3$ & $120 \pm 10$ \\
\hline
\end{tabular}

\subsection{Analysis of SAXS Data for the Ground and the Light-Activated States of SML-PYP}

To investigate the structure of the ground and the light-activated states of SML-PYP, we performed the small-angle X-ray scattering (SAXS) experiment with and without continuous blue light irradiation (Figure 3a,b). PYP is a highly soluble protein [5] and even after the incorporation of BM(PEG) 2 the 
solubility of the protein was maintained. To rule out the distortion of X-ray scattering curve originated from the aggregated species, the solution was cautiously centrifuged at 10,000 $\mathrm{g}$ for $10 \mathrm{~min}$ prior to performing the SAXS measurements. As shown in Figure S6, the Guinier plot of the original SAXS data shows an excellent linearity, which implies that the scattering signal in the small-angle region is free from aggregation. Radius of gyration $(R g)$ values for the dark and light states of SML-PYP were determined as 15.3 and $15.4 \AA$, respectively, as determined with the PRIMUS program of the ATSAS software package [18-20]. We assumed full conversion from dark state to light state because we estimated the conversion rate to be over $97.5 \%$ based on the UV-vis spectrum under continuous light-emitting diode (LED) illumination (see Figure S7). The Rg value of the ground state for SML-PYP is similar to that of the light-activated state for wild-type PYP (15.6 $⿱$ ) rather than that of the dark state for wild-type PYP. Since the size of the small-molecule linker itself is too small to affect the $R g$ value, this increased $R g$ value might be due to the conformational change of the protein body, and suggests that, compared to that of wild-type PYP, the ground state of SML-PYP has a more swollen structure, which possibly has the $\mathrm{N}$-terminal protrusion due to the instability arising from the linker conjugation. The $\mathrm{Rg}$ value of light-activated state for SML-PYP is slightly larger than that of the ground state for SML-PYP. The difference between the $R g$ values of the light and ground states of SML-PYP is only $0.13 \AA$, which is much smaller than that of the wild-type PYP $(1.2 \AA)$, indicating that the structural change upon light irradiation of SML-PYP is indeed restricted compared to that of wild-type PYP, fulfilling our goal of regulating the degree of structural change by small-molecule linkage.
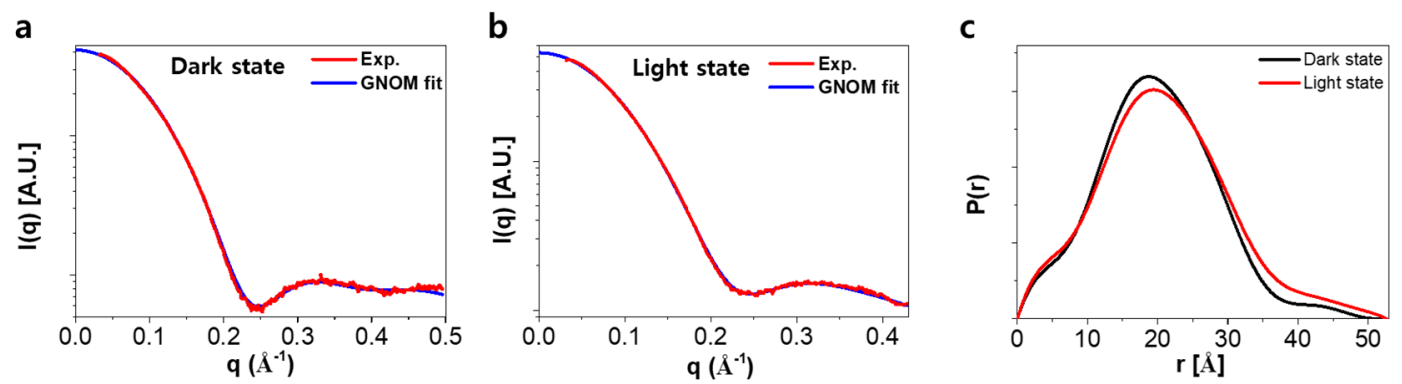

Figure 3. SAXS (red) and GNOM fit results (blue) for BM(PEG) ${ }_{2}$-incorporated PYP (SML-PYP) in the dark (a) and the light (b) states. (c) Pair distribution, $P(r)$, of SML-PYP with (red) and without (black) exposure to light. $P(r)$ functions show that the light state of SML-PYP has a larger Rmax value. A. U. stands for arbitrary unit.

\subsection{Molecular Shape Reconstruction from the SAXS Data}

Prior to shape reconstruction, the pair distribution functions, $P(r)$, of the dark and light states for SML-PYP were obtained using the GNOM program (Figure 3c) [21]. $P(r)$ of the dark state shows the main distribution at $\sim 20 \AA$ and a minor distribution reaching $50 \AA . P(r)$ of the light state shows a similar main distribution with slightly less population compared to that of the dark state and a minor distribution reaching $53 \AA$. The ratio between the main and minor distributions slightly changed between the light and dark states, indicating that blue light irradiation resulted in restricted structural changes of SML-PYP, probably because of slight unfolding.

Next, we performed shape reconstruction using the DAMMIF program and GNOM data to estimate the shapes of the dark and light states of SML-PYP (Figure S8) [22]. The reconstructed shapes of the dark and light states of SML-PYP were obtained for each state (Figure 4a-c). The light state structure is superimposed on the dark state structure of SML-PYP in Figure 4c. The light state shape of SML-PYP is slightly more anisotropic and elongated along the long axis compared to the dark state shape, but the degree of change is small. 
a

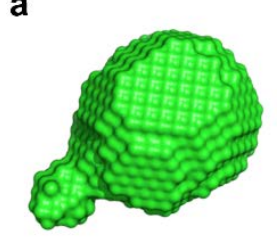

d

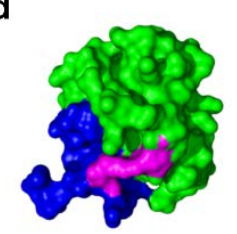

g

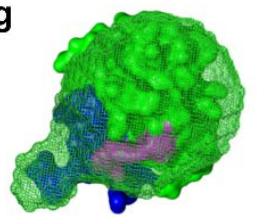

b

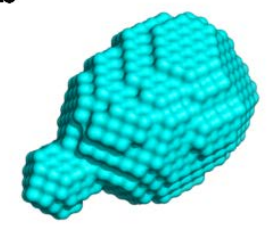

e

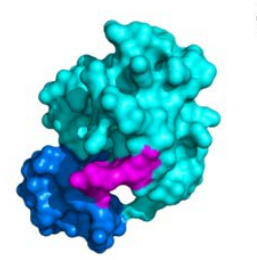

h

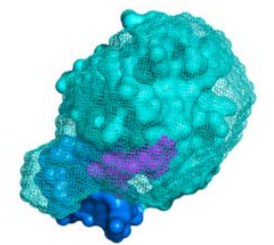

c

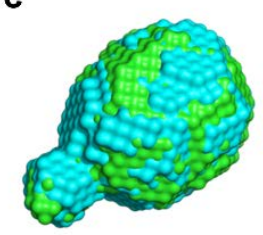

f

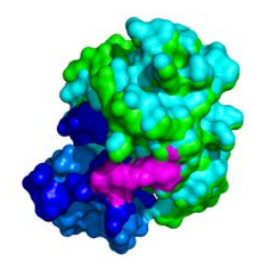

Figure 4. Molecular structures modeled from the low-resolution shape reconstruction and the experiment-restrained rigid-body MD simulation. $(\mathbf{a}-\mathbf{c})$ Low-resolution shape reconstruction of SML-PYP in the dark (a) and light (b) states, and the superimposed image (c) of DAMMIF filtered results. (d-f) Structural representation of SML-PYP in the dark (d) and light (e) states from the experiment-restrained rigid-body MD simulation, and the resulting superimposed image (f). (g,h) Comparison of structures from the low-resolution shape reconstruction (mesh) and the experiment-restrained rigid-body MD simulation (sphere-dot) of SML-PYP in the dark (g) and light (h) states. Blue and marine colors indicate 27 amino acids in the N-terminal region of SML-PYP in the dark and light states, respectively. Green and cyan colors indicate core region of SML-PYP in the dark and light states. Magenta color indicates the $\mathrm{BM}(\mathrm{PEG})_{2}$ molecule.

\subsection{Experiment-Restrained Rigid-Body MD Simulation}

To more precisely define which part of the protein is responsible for the difference between SML-PYP and wild-type PYP, we also performed the experiment-restrained rigid-body MD simulations and thus determined the structure of the dark and light states (Figure S9 and Figure $4 d-f$ ) $[7,23]$. In this simulation, we used a q-value as large as $0.5 \AA^{-1}$ and produced a model based on all available data to facilitate higher resolution of the protein shape. As shown in Figure 4d, the dark state structure of SML-PYP shows the protrusion of the N-terminus, as expected in the comparison of $R g$ values and reconstructed shapes between SML-PYP and wild-type PYP (Figure 4g). Moreover, the light state structure of SML-PYP shows slight elongation along the long axis compared to the dark state, similar to the result obtained with the shape reconstruction (Figure $4 \mathrm{~h}$ ). The degree of the elongation due to the dark to light conversion upon blue light irradiation for SML-PYP is not as significant as that for wild-type PYP, which is consistent with the relatively small Rg value changes of SML-PYP. The mutated positions of the 7th and 91st residues are located on the N-terminus and core, respectively, and the intramolecular conjugation using a linker molecule probably constrains the $\mathrm{N}$-terminus structural changes of SML-PYP compared to those of the wild-type PYP. This scenario is schematically represented in Figure 5.

\section{Discussion}

It is important to understand the mechanical and structural changes in proteins to achieve fine control of structural changes in protein-based nanomachines [24]. To do so, we investigated a new method which is the incorporation of a small-molecule linker into the protein. This work may serve as the starting point for the development of strategies to regulate protein structural changes. 
The CD spectrum of the mutant PYP (G7C-M91C PYP) is quite similar to that of wild-type PYP, whereas the CD spectrum of SML-PYP indicates that its $\alpha$-helix content is moderately reduced (Figure S5). The SAXS data show an increase of Rg value in the ground state (pG) of SML-PYP by $0.9 \AA$ compared with that of wild-type PYP, which corresponds to an approximate volume increase of $5500 \AA^{3}$. The volume of the six residues in the N-terminal region would be about $147 \AA^{3}$ plus the volume of the hydration shell and thus this alone cannot account for the $0.9 \AA$ increase in $R g$ value. On the other hand, the environment around the chromophore region of SML-PYP is expected to be well conserved and similar to that of the wild-type based on the similarity of the UV-vis spectra (see Figure S2). The reduced $\alpha$-helix content, the increased $\mathrm{Rg}$ value and the well-conserved chromophore environment all together suggest that SML-PYP has a swollen conformation with a well-conserved core. In accordance with this possibility, the molecular shape reconstructed from the SAXS data shows a partial protrusion in the ground state of SML-PYP (Figure 4a). The molecular origin of the partial protrusion can be identified by comparing the shape and the structure from the experiment-restrained rigid-body MD simulation (Figure $4 \mathrm{~d}$ ). The protrusion of the reconstructed shape well matches the $\mathrm{N}$-terminal region in the structure from the experiment-restrained rigid-body MD simulation, confirming that the $\mathrm{N}$-terminal region is indeed responsible for the protrusion. This result, which we did not anticipate when we had chosen the cross-linking positions and the small-molecule linker, shows that the overall shape of the protein molecule can be easily affected by the small-molecule linker. We note that, because the experiment-restrained rigid-body MD simulation yields only a single protein structure, the resulting structure cannot properly represent the ensemble with the conformational heterogeneity. Nonetheless, judging from the good agreement between the dummy-atom modeled shape and the structure from the experiment-restrained rigid-body MD simulation shown in Figure 4, we suggest that the latter may represent a protein conformation of the microstate included in the ensemble.

A study using time-resolved X-ray solution scattering reports large changes in the $R g$ value from 14.4 to $15.6 \AA$ for wild-type PYP upon blue light irradiation [11]. However, in the case of SML-PYP, the $R g$ value changed from 15.3 to $15.5 \AA$ upon blue light irradiation. The structure of the light state determined by the shape reconstruction and the experiment-restrained rigid-body MD simulation also shows only minor N-terminal structural changes including a slight elongation of the protein along the long axis, whereas wild-type PYP shows dramatic structural changes accompanied by N-terminal protrusion. The reduced structural changes induced by the small-molecule linker demonstrate that our designed strategy works. The marginal change of N-terminal protrusion of SML-PYP may originate from the cross-linking incorporated in PYP that significantly restricts the mechanical motion of the $\mathrm{N}$-terminus. Thus, we demonstrate that the structural changes in a protein can be restricted by introducing a small-molecule linker (Figure 5 and Figure S10). Furthermore, this result is additional evidence that the structural changes of the N-terminus are essential for the formation of the light state of wild-type PYP because our results confirm cross-linking the N-terminus and the core of PYP restricts the photoinduced protrusion of the $\mathrm{N}$-terminal region. In other words, this result confirms the suggestion by previous studies $[11,12]$ that the $\mathrm{N}$-terminus protrusion is the major structural change in the late intermediate stage.

The TA results show that mutation and cross-linking affect the kinetics of the formation of the light state, $\mathrm{pB}_{2}$, in SML-PYP. The time constant of the $\mathrm{pB}_{2}$ formation for SML-PYP is two times faster $(480 \mu \mathrm{s})$ than that for wild-type PYP $(920 \mu \mathrm{s})$. This can be rationalized by considering the structural aspect of SML-PYP, i.e. the PG structure of SML-PYP is a swollen conformation relative to that of wild-type. This pre-swollen conformation in $\mathrm{pG}$ may facilitate the conversion to $\mathrm{pB}_{2}$, resulting in the modest acceleration of the $\mathrm{pB}_{1} \rightarrow \mathrm{pB}_{2}$ transition. 
a

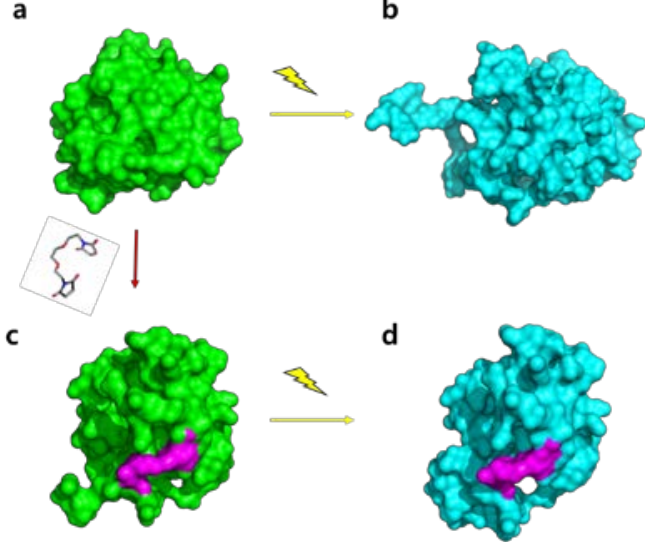

Figure 5. Schematic illustration of the structural changes in PYP induced by small-molecule binding $(\mathbf{a} \rightarrow \mathbf{c})$ and blue light irradiation $(\mathbf{a} \rightarrow \mathbf{b}, \mathbf{c} \rightarrow \mathbf{d})$. (a) The dark state of wild-type PYP. (b) The light state of wild-type PYP. (c) The dark state of SML-PYP. (d) The light state of SML-PYP. The small-molecule linker is shown in magenta. Wild-type PYP dark and light structures are from PDB (protein data bank) ID, 2PHY and 2KX6, respectively, and the dark and light structures of SML-PYP are from this work.

\section{Materials and Methods}

\subsection{Preparation of PYP with Blocked N-terminal Movement}

We tried to prepare various constructs of PYP, with different mutated pairs of amino acid residues: 7 th and 91st, 13th and 113th, and 7th and 113th residues. The pair of amino acids were mutated into cysteines, with a thiol (-SH) side chain. The mutants were tested for the potential of the thiol groups of the cysteines to conjugate with various linkers, such as bismaleimidoethane (BMOE), 1,8-bismaleimidodiethyleneglycol (BM(PEG) $)$, and 1,4-bismaleimidobutane (BMB). The modified PYP was purified using previously reported methods (see Supplementary Materials) [25]. After incubating with the linker, the PYPs were analyzed by MALDI/TOF and Thiol Fluorescent Detection Kit (Invitrogen ${ }^{\mathrm{TM}}$ ) to confirm the degree of conjugation. CD spectra were also measured to check structural stability (Figure S5).

\subsection{Transient Absorption (TA) Measurement}

TA measurement was performed by following previously reported procedures [26-29]. A pump beam $(460 \mathrm{~nm})$ was produced by an optical parametric oscillator (LT-2214-PC, LOTIS, Minsk, Belarus) pumped by a third harmonics (355 nm) of an Nd:YAG laser (NL 301G, EKSPLA, Vilnius, Lithuania). A probe beam was generated by a continuous wave $250 \mathrm{~W}$ Xe-lamp (66902, Newport, Irvine, CA, USA). The probe light and the pump laser beam were intersected on a sample position at an acute angle to each other to maximize the overlap area. TA signal was detected by a combination of a spectrometer (SpectraPro 2300i, Princeton Instrument, Acton, MA, USA) and a detector. We used two types of detectors: an ICCD (iStar, Andor, Belfast, United Kingdom) was used for TA spectra measurements and a photomultiplier tube (PMT) connected with a digital oscilloscope (TDS 3052B, Tektronix, Beaverton, OR, USA) was used for temporal profile measurements. To improve the signal-to-noise ratio, the TA spectra and the temporal profiles were averaged 200 times and 320 times, respectively. The concentrations of PYPs were adjusted to be about $70 \mu \mathrm{M}$. The sample solution was injected and flowed by using a syringe pump and a $2 \mathrm{~mm}$ quartz flow cell. The repetition rate of the pump beam was 1 or $0.5 \mathrm{~Hz}$.

\section{3. $S A X S$}

X-ray scattering data as a function of scattering vector $(q)$ were collected at the 4C SAXS II beamline at Pohang Light Source (PLS-II) in Korea. Monochromatic X-ray pulse with the energy of 
$16.90 \mathrm{keV}$ was used for data collection. Static X-ray scattering patterns were recorded using a MarCCD detector (Rayonix, Evanston, IL, USA). The sample-to-detector distance was set at $1 \mathrm{~m}$. The $q$ ranges of the data for dark and light states were $0.03-0.50 \AA^{-1}$ and $0.03-0.43 \AA^{-1}$, respectively. The protein was dissolved in the buffer solution of $20 \mathrm{mM}$ Tris ( $\mathrm{pH} 7.0$ ) with $20 \mathrm{mM} \mathrm{NaCl}$ and the concentration of the protein was $10 \mathrm{mg} / \mathrm{mL}$. The sample solution was centrifuged at 10,000 $\mathrm{g}$ for $10 \mathrm{~min}$ to remove unwanted aggregated particles and only the supernatant was used for the measurement prior to performing the SAXS measurements. To populate the light state, the protein sample was continuously irradiated by focused blue LED light during the measurement. To avoid any damages induced from X-ray and optical lights, the X-ray scattering curves of the SML-PYP were obtained by employing a flow cell system (Figure S11). The PYP sample solution was injected into a capillary at $100 \mu \mathrm{L} / \mathrm{min}$. The capillary was $1.0 \mathrm{~mm}$ thick, and the $\mathrm{X}$-ray exposure time was $60 \mathrm{~s}$. To confirm photo-conversion ratio to $\mathrm{pB}_{2}$, we measured a UV-vis spectrum using the same setup (Figure $\mathrm{S} 7$ ).

\subsection{SAXS Analysis and Molecular Shape Reconstruction}

The SAXS data were processed and analyzed using the ATSAS package (http://www.emblhamburg.de/biosaxs/software.html). One-dimensional scattering data, $I(q)$, were obtained by azimuthal averaging. Scattering intensities for the pure buffer solution were measured by a separate experiment to determine the background intensity, and the $I(q)$ were extrapolated to $q=0$ by GNOM program. The $\mathrm{Rg}$ value and the pair distribution function, $P(r)$, were calculated through indirect Fourier transformation [21]. The $q$ and real space range for the dark state of SML-PYP are $0.05-0.50 \AA^{-1}$ and $0.00-50.06 \AA$, respectively, and those for the light state of SML-PYP are $0.05-0.40 \AA^{-1}$ and 0.00-54.16 $\AA$, respectively. The maximum dimension (Dmax), which is the maximum distance between scatterers was determined from PRIMUS at ATSAS. Using DAMMIF program [22], ab-initio shape reconstruction for the X-ray data with the q range of $0.001-0.29 \AA^{-1}$ and the optimal $P(r)$ function was performed to reconstruct the shape of the protein. This shape reconstruction was repeated nineteen times for each data. Representative structures were generated by superimposing each structure determined from DAMMIF process result onto a template structure using DAMSEL and DAMSUP. Nineteen low-resolution dummy atomic models were averaged using DAMAVER [30]. DAMFILT was used to obtain a representative structure with high-probability densities. The reconstructed shape was compared with those of models obtained from the experiment-restrained rigid-body MD simulations to determine the conformational changes induced by blue light irradiation at the atomic level. The reconstructed shapes and experiment-restrained rigid-body MD simulation results were superimposed using SUPCOMB [31].

\subsection{SAXS Curve Analysis with the Experiment-Restrained Rigid-Body MD Simulation}

Structures and shape of SML-PYP in the dark and light state were obtained from the experiment-restrained rigid-body MD simulations (see Supplementary Materials). We used the crystal structure of PYP (PDB ID: 2PHY) and manually attached it with the molecular linker for use as the initial structure in the experiment-restrained rigid-body MD simulation. In this simulation, 2-3 amino acids in the loop region and 4-12 amino acids in the alpha helix and beta sheet were grouped as a single rigid body to reduce the number of parameters and calculation time for the simulation. In total, $\sim 29$ rigid bodies were used in the experiment-restrained rigid-body MD simulations. The CRYSOL program [32] was used to calculate the theoretical X-ray scattering curve of the refined structure in the experiment-restrained rigid-body MD simulations. In this simulation, we minimized the value of a target function that represents the discrepancy between the GNOM curves originated from experiment and the theoretical scattering curves on the q range of $0-0.43 \AA^{-1}$. In the target function, the van der Waals forces between rigid bodies and the correction terms for bond lengths were also considered to minimize chemical instability. Then, we fitted the experimental curve by changing the CRYSOL parameters, such as the contrast in the hydration shell, the radius of the atomic group, excluded volume, and scaling factor between the experimental and theoretical results. For the fitting, we 
employed the MINUIT program in ROOT [33] from CERN. The experiment-restrained rigid-body MD simulation and the fit of the CRYSOL parameter were performed iteratively until the smallest chi-square value was achieved. Finally, we determined the best model exhibiting a good agreement between the experimental SAXS curve and the theoretical scattering curve.

\section{Conclusions}

SAXS and TA results show that cross-linking between the N-terminus and the core of PYP with $\mathrm{BM}(\mathrm{PEG})_{2}$ may constrain the N-terminal movement of PYP. SML-PYP slightly increased in size, including elongation along the long axis upon blue light irradiation, whereas wild-type PYP exhibited large structural changes accompanied by N-terminal protrusion. We demonstrate that the incorporation of a small-molecule linker can be used for regulating the structural dynamics of the protein. The ability to regulate the mechanical motions with cross-linking may provide a new strategy for the mechanical manipulation of protein nanotechnology and lead to novel nanomachines for a variety of applications.

Supplementary Materials: Supplementary Materials can be found at http:/ / www.mdpi.com/1422-0067/19/12/ 3714/s1. Figure S1: The 7th (glycine) and 91st (methionine) of PYP were mutated to cysteines.; Figure S2: UV-vis spectra of wild-type PYP (black) and BM(PEG)2-incorporated PYP (SML-PYP, red).; Figure S3: MALDI/TOF result for wild-type PYP (black, $14.02 \mathrm{kDa}$ ) and BM(PEG)2-incorporated PYP (SML-PYP) (red, 14.36 kDa).; Figure S4: Thiol Fluorescent Detection Kit (Invitrogen ${ }^{\mathrm{TM}}$ ) result.; Figure S5: Circular dichroism spectra of wild-type PYP, G7C-M91C mutant, and SMLPYP.; Figure S6: Guinier region of SAXS data for BM(PEG)2-incorporated PYP (SML-PYP) in the dark state.; Figure S7: UV-vis spectra of PYP with/without continuous LED illumination.; Figure S8: Comparison of DAMMIF-fit curves (black) and the experiment curves (red) from static SAXS data for SML-PYP in dark (a) and light (b) states for low-resolution shape reconstruction.; Figure S9: GNOM curve from static SAXS data (red) and the fit curve from experiment restrained rigid-body molecular dynamics simulation (black) for SML-PYP in dark (a) and light (b) states.; Figure S10: Protein structures refined from the experiment-restrained rigid-body MD simulation and previous reported wild-type PYP and pB2 structure.; Figure S11: SAXS setup for SML-PYP in the light state.

Author Contributions: Conceptualization, H.I. and Y.K. (Youngmin Kim); Methodology, Y.K. (Youngmin Kim) and C.Y.; Provision, Y.K. (Youngmin Kim), C.Y., T.W.K., K.T., and Y.K. (Yonggwan Kim); Investigation, Y.K. (Youngmin Kim), C.Y., T.W.K., K.T., and Y.K. (Yonggwan Kim); Writing-Original Draft Preparation, Y.K. (Youngmin Kim); Writing-Review and Editing, H.I., Y.K. (Youngmin Kim), T.W.K., C.Y., and K.T.; Visualization, Y.K. (Youngmin Kim) and C.Y.; Supervision, H.I.; Project Administration, H.I.; and Funding Acquisition, H.I.

Funding: This study was supported by Institute for Basic Science (IBS-R004).

Acknowledgments: We acknowledge extensive support from Kyeong Sik Jin of 4C SAXS beamline at Pohang Light Source. We thank Byoungkook Kim in KAIST Analysis center for Research Advancement (KARA) for circular dichroism experiments.

Conflicts of Interest: The authors declare no conflicts of interest.

\section{Abbreviations}

$\begin{array}{ll}\text { PYP } & \text { Photoactive yellow protein } \\ \text { SML } & \text { Small-molecule linker } \\ \text { BM(PEG) } 2 & \text { 1,8-Bismaleimidodiethyleneglycol } \\ \text { SML-PYP } & \text { Cross-linker (BM(PEG) })_{2} \text {-conjugated PYP } \\ \text { MD } & \text { Molecular dynamics } \\ \text { CD } & \text { Circular dichroism } \\ \text { Rg } & \text { Radius of gyration } \\ \text { SAXS } & \text { Small-angle X-ray solution scattering } \\ \text { MALDI/TOF } & \text { Matrix-assisted laser desorption/ionization } \\ \text { TA } & \text { time-of-flight } \\ & \text { Transient absorption }\end{array}$




\section{References}

1. Benkovic, S.J.; Raney, K.D. Mechanisms: Molecular machines. Curr. Opin. Chem. Biol. 2011, 15, 577-579. [CrossRef] [PubMed]

2. Zhang, L.; Marcos, V.; Leigh, D.A. Molecular machines with bio-inspired mechanisms. Proc. Natl. Acad. Sci. USA 2018, 115, 9397-9404. [CrossRef] [PubMed]

3. Ali, A.M.; Woolley, G.A. The effect of azobenzene cross-linker position on the degree of helical peptide photo-control. Org. Biomol. Chem. 2013, 11, 5325-5331. [CrossRef] [PubMed]

4. Woolley, G.A. Protein machines: An open and shut cage. Nat. Nanotechnol. 2013, 8, 892-893. [CrossRef] [PubMed]

5. Imamoto, Y.; Ito, T.; Kataoka, M.; Tokunaga, F. Reconstitution photoactive yellow protein from apoprotein and P-coumaric acid-derivatives. FEBS Lett. 1995, 374, 157-160. [CrossRef]

6. Lee, K.; Kim, Y.; Jung, J.; Ihee, H.; Park, Y. Measurements of complex refractive index change of photoactive yellow protein over a wide wavelength range using hyperspectral quantitative phase imaging. Sci. Rep. 2018, 8, 3064. [CrossRef] [PubMed]

7. Kim, Y.; Ganesan, P.; Jo, J.; Kim, S.O.; Thamilselvan, K.; Ihee, H. Chromophore-removal-induced conformational change in photoactive yellow protein determined through spectroscopic and $X$-ray solution scattering studies. J. Phys. Chem. B 2018, 122, 4513-4520. [CrossRef] [PubMed]

8. Yang, C.; Kim, T.W.; Kim, Y.; Choi, J.; Lee, S.J.; Ihee, H. Kinetics of the E46Q mutant of photoactive yellow protein investigated by transient grating spectroscopy. Chem. Phys. Lett. 2017, 683, 262-267. [CrossRef]

9. Kumar, A.; Burns, D.C.; Al-Abdul-Wahid, M.S.; Woolley, G.A. A Circularly permuted photoactive yellow protein as a scaffold for photoswitch design. Biochemistry 2013, 52, 3320-3331. [CrossRef] [PubMed]

10. Jung, Y.O.; Lee, J.H.; Kim, J.; Schmidt, M.; Moffat, K.; Srajer, V.; Thee, H. Volume-conserving trans-cis isomerization pathways in photoactive yellow protein visualized by picosecond X-ray crystallography. Nat. Chem. 2013, 5, 212-220. [CrossRef] [PubMed]

11. Kim, T.W.; Lee, J.H.; Choi, J.; Kim, K.H.; van Wilderen, L.J.; Guerin, L.; Kim, Y.; Jung, Y.O.; Yang, C.; Kim, J.; et al. Protein structural dynamics of photoactive yellow protein in solution revealed by pump-probe $\mathrm{X}$-ray solution scattering. J. Am. Chem. Soc. 2012, 134, 3145-3153. [CrossRef] [PubMed]

12. Ramachandran, P.L.; Lovett, J.E.; Carl, P.J.; Cammarata, M.; Lee, J.H.; Jung, Y.O.; Ihee, H.; Timmel, C.R.; van Thor, J.J. The short-lived signaling state of the photoactive yellow protein photoreceptor revealed by combined structural probes. J. Am. Chem. Soc. 2011, 133, 9395-9404. [CrossRef] [PubMed]

13. Imamoto, Y.; Kataoka, M.; Tokunaga, F.; Asahi, T.; Masuhara, H. Primary photoreaction of photoactive yellow protein studied by subpicosecond-nanosecond spectroscopy. Biochemistry 2001, 40, 6047-6052. [CrossRef] [PubMed]

14. Takeshita, K.; Imamoto, Y.; Kataoka, M.; Mihara, K.; Tokunaga, F.; Terazima, M. Structural change of site-directed mutants of PYP: New dynamics during pR state. Biophys. J. 2002, 83, 1567-1577. [CrossRef]

15. Losi, A.; Gensch, T.; van der Horst, M.A.; Hellingwerf, K.J.; Braslavsky, S.E. Hydrogen-bond network probed by time-resolved optoacoustic spectroscopy: Photoactive yellow protein and the effect of e46q and e46a mutations. Phys. Chem. Chem. Phys. 2005, 7, 2229-2236. [CrossRef] [PubMed]

16. Ihee, H.; Rajagopal, S.; Srajer, V.; Pahl, R.; Anderson, S.; Schmidt, M.; Schotte, F.; Anfinrud, P.A.; Wulff, M.; Moffat, K. Visualizing reaction pathways in photoactive yellow protein from nanoseconds to seconds. Proc. Natl. Acad. Sci. USA 2005, 102, 7145-7150. [CrossRef] [PubMed]

17. Yeremenko, S.; van Stokkum, I.H.M.; Moffat, K.; Hellingwerf, K.J. Influence of the crystalline state on photoinduced dynamics of photoactive yellow protein studied by ultraviolet-visible transient absorption spectroscopy. Biophys. J. 2006, 90, 4224-4235. [CrossRef] [PubMed]

18. Svergun, D.I. Small-angle X-ray and neutron scattering as a tool for structural systems biology. Biol. Chem. 2010, 391, 737-743. [CrossRef] [PubMed]

19. Jacques, D.A.; Trewhella, J. Small-angle scattering for structural biology-Expanding the frontier while avoiding the pitfalls. Protein Sci. 2010, 19, 642-657. [CrossRef] [PubMed]

20. Putnam, C.D.; Hammel, M.; Hura, G.L.; Tainer, J.A. X-ray solution scattering (SAXS) combined with crystallography and computation: Defining accurate macromolecular structures, conformations and assemblies in solution. Q. Rev. Biophys. 2007, 40, 191-285. [CrossRef] [PubMed] 
21. Svergun, D.I. Determination of the regularization parameter in indirect-transform methods using perceptual criteria. J. Appl. Crystallogr. 1992, 25, 495-503. [CrossRef]

22. Franke, D.; Svergun, D.I. DAMMIF, a program for rapid ab-initio shape determination in small-angle scattering. J. Appl. Crystallogr. 2009, 42, 342-346. [CrossRef] [PubMed]

23. Ahn, S.; Kim, K.H.; Kim, Y.; Kim, J.; Thee, H. Protein tertiary structural changes visualized by time-resolved X-ray solution scattering. J. Phys. Chem. B 2009, 113, 13131-13133. [CrossRef] [PubMed]

24. Amrute-Nayak, M.; Diensthuber, R.P.; Steffen, W.; Kathmann, D.; Hartmann, F.K.; Fedorov, R.; Urbanke, C.; Manstein, D.J.; Brenner, B.; Tsiavaliaris, G. Targeted optimization of a protein nanomachine for operation in biohybrid devices. Angew. Chem. Int. Ed. 2010, 49, 312-316. [CrossRef] [PubMed]

25. Kim, Y.; Ganesan, P.; Ihee, H. High-throughput instant quantification of protein expression and purity based on photoactive yellow protein turn off/on label. Protein Sci. 2013, 22, 1109-1117. [CrossRef] [PubMed]

26. Choi, J.; Jung, Y.O.; Lee, J.H.; Yang, C.; Kim, B.; Ihee, H. Folding dynamics of ferrocytochrome c in a denaturant-free environment probed by transient grating spectroscopy. ChemPhysChem 2008, 9, 2708-2714. [CrossRef] [PubMed]

27. Choi, J.; Muniyappan, S.; Wallis, J.T.; Royer, W.E.; Ihee, H. Protein conformational dynamics of homodimeric hemoglobin revealed by combined time-resolved spectroscopic probes. ChemPhysChem 2010, 11, 109-114. [CrossRef] [PubMed]

28. Choi, J.; Yang, C.; Kim, J.; Ihee, H. Protein folding dynamics of cytochrome c seen by transient grating and transient absorption spectroscopies. J. Phys. Chem. B 2011, 115, 3127-3135. [CrossRef] [PubMed]

29. Yang, C.; Choi, J.; Ihee, H. The time scale of the quaternary structural changes in hemoglobin revealed using the transient grating technique. Phys. Chem. Chem. Phys. 2015, 17, 22571-22575. [CrossRef] [PubMed]

30. Volkov, V.V.; Svergun, D.I. Uniqueness of ab initio shape determination in small-angle scattering. J. Appl. Crystallogr. 2003, 36, 860-864. [CrossRef]

31. Kozin, M.B.; Svergun, D.I. Automated matching of high- and low-resolution structural models. J. Appl. Crystallogr. 2001, 34, 33-41. [CrossRef]

32. Svergun, D.; Barberato, C.; Koch, M.H.J. CRYSOL-A program to evaluate x-ray solution scattering of biological macromolecules from atomic coordinates. J. Appl. Crystallogr. 1995, 28, 768-773. [CrossRef]

33. James, F.; Roos, M. Minuit-system for function minimization and analysis of parameter errors and correlations. Comput. Phys. Commun. 1975, 10, 343-367. [CrossRef] 